\title{
Comparative Evaluation of Wear Resistance Among Cad-Cam and Resin Interim Restoration - an in Vitro Study
}

\author{
Meghana Reddy J1', Ashok V², Kiran Kumar ${ }^{3}$ and Dhanraj Ganapathy ${ }^{4}$ \\ ${ }^{1}$ Saveetha Dental College and Hospitals, Saveetha Institute of Medical and \\ Technical Sciences (SIMATS), Saveetha University, Chennai, India \\ Professor and Head (Admin), Department of Prosthodontics Saveetha Dental \\ College and Hospitals Saveetha Institute of Medical and Technical Sciences, \\ Saveetha University Chennai, India \\ ${ }^{3}$ Senior Lecturer Department of Prosthodontics Saveetha Dental College and \\ Hospitals Saveetha Institute of Medical and Technical Sciences, Saveetha \\ University Chennai, India \\ ${ }^{4}$ Professor and Head (Academics), Department of Prosthodontics Saveetha Dental \\ College and Hospitals Saveetha Institute of Medical and Technical Sciences, \\ Saveetha University Chennai, India
}

\section{ABSTRACT}

The most commonly used material in dentistry is Poly methyl methacrylate (PMMA) which has excellent properties like physical and mechanical properties but this material is rarely used in pure form as it has relatively poor wear resistance and heat resistance. The ideal properties of fixed partial denture (FPD) base material should have superior surface texture and mechanical properties. The wear resistance of heat cure provisional and CAD-CAM provisional vary greatly based on their properties. The aim of this study was to comparatively evaluate the wear resistance in heat cure provisional and cad-cam provisional fixed partial denture. Eight samples each of heat cure provisional and CAD-CAM milled FPD are prepared. The samples are then run for 8 hours in a brushing simulator with a toothpaste to check for its wear resistance .The pre test and post test measurement of its wear resistance is done using laser scan. The wear resistance is more for the CAD-CAM provisional FPD as compared to the heat cure provisional FPD. Pre treatment and post treatment cuspal thickness shows a vast difference in heat cure provisional. However the pre treatment and post treatment cuspal thickness reduction is minimal in CAD CAM provisional FPD. CAD CAM provisional FPD showed better wear resistance than heat cured provisional FPD. Hence CAD CAM prosthesis should be preferred over the heat cured FPD.

KEY WORDS: WEAR RESISTANCE, HEAT CURE PROVISIONAL, CAD-CAM PROVISIONAL.

\section{ARTICLE INFORMATION}

*Corresponding Author: ashok@saveetha.com

Received 4th Aug 2020 Accepted after revision 26th Sep 2020

Print ISSN: 0974-6455 Online ISSN: 2321-4007 CODEN: BBRCBA

Thomson Reuters ISI Web of Science Clarivate Analytics USA and Crossref Indexed Journal

$$
\begin{aligned}
& \text { Clarivate } \\
& \text { Analytics }
\end{aligned}
$$




\section{INTRODUCTION}

The success of the restoration in fixed prosthodontics depends on three main factors that are biological factors, mechanical factors, and aesthetic factors. Different materials have been tried in restoring the missing tooth. The choice of the material selection depends on the esthetics, available interocclusal space, number of missing teeth, cost and the status of the abutment tooth (Baba, 2016).

Ideally FPD (fixed partial denture) base material should have superior surface texture and mechanical properties. In 1936 Walter Wright introduced acrylic resin material called polymethylmethacrylate (PMMA) to the market (Keyf, Uzun and Mutlu, 2003; Baba, 2016). This material became one of the most popularly used denture base materials due to its ease of handling and manipulation of the material, it is less toxic, has good aesthetic results, shows adequate strength, easily repaired, the stability in the oral cavity was better when compared with other materials, low solubility, reasonable cost, and low water absorption (Kim and Watts, 2004). However, the mechanical properties of PMMA have been considered inadequate (Vallittu, 1993, 1999). Among the common drawbacks of PMMA are dimensional changes, susceptibility to fracture, residual monomers, and increased risk of denture-associated infections (Vallittu, Ruyter and Ekstrand, 1998). In addition to this the possibility of surface and subsurface voids, which can not only jeopardize the mechanical properties of the processed denture but also compromise esthetic and hygienic results (Hazelton et al., 1995; Vallittu, Ruyter and Ekstrand, 1998).

Surface characteristics of acrylic dentures such as roughness, hardness, and wettability have been reported to be key players in denture-associated stomatitis (John et al., 2015). Surface roughness has been described as irregularities that characterizes a surface and has its influence on wetting, quality of adhesion, and brightness of that surface. Rough surfaces generally tend to induce halitosis and are considered more vulnerable to discoloration than smooth surfaces, thereby reducing patient esthetics and comfort (Cardoso et al., 2008).

As microbial adhesion and colonization usually occur on non shedding surfaces (Lewinstein et al., 2003), dental prostheses need to have smooth surfaces to minimize the retention of plaque and microorganisms (Vallittu, Ruyter and Ekstrand, 1998; Cardoso et al., 2008). To decrease the accumulation and colonization of microorganisms, the surface roughness of dental prostheses should not exceed a threshold of $0.2 \mu \mathrm{m}$ (Scotti, Mascellani and Forniti, 1997; Cai et al., 2011; Ayman, 2017). Studies reported that a $0.2 \mu \mathrm{m}$ roughness threshold can be achieved by common laboratory and chairside finishing and polishing procedures.Therefore, adequate finishing and polishing of dental prostheses, including dentures, are mandatory to minimize prosthesis surface roughness (Scotti, Mascellani and Forniti, 1997).[16]
Computer-aided design/computer-aided manufacturing (CAD/CAM) techniques haveexpanded recently to embrace the fabrication of complete dentures, record bases, immediate dentures, and implant-supported overdentures in two clinical appointments. As CAD/ CAM dentures are milled from pre-polymerized PMMA billets that are polymerized under high temperatures and pressure values, $\mathrm{CAD} / \mathrm{CAM}$ dentures are reported to be less porous, and consequently, less likely to harbor virulent microorganisms such as Candida albicans, which will be less able to adhere to the surface of digital dentures (Ruyter, Nilner and Moller, 1987; Wassell et al., 2002; Diaz-Arnold et al., 2008).

The material of choice for the fixed restoration in compromised abutments and in immediate implant loading is the polymer resin. Polymethyl methacrylate (PMMA) is the most commonly used polymer as a removable prosthetic restorative material due to its excellent physical and mechanical properties. Due to its poor wear resistance and heat resistance, it is not used as a permanent fixed restorative material (Demir et al., 2006; Cai et al., 2011).

In polymer tribology, wear mechanism includes abrasive, adhesive and fatigue wear. Abrasive wear is a material loss phenomenon due to movement of hard particles on the friction surface. Adhesive wear, it is the adhesion and tearing micro materials during friction. Fatigue wear is the peeling of material under repeated action of high compressive stress (Gu et al., 2019).

Heat treatment is considered as one of the most effective ways to improve the mechanical properties of polymer materials (Ovsianikov et al., 2011; Gu et al., 2019). Heat cured PMMA resin is the most widely used material for removable prosthesis. This material is mainly used due to its physical, chemical properties, easy to process and reasonable cost. However, there are few disadvantages like release of residual methyl methacrylate (MMA) which affects the dimensional stability of the material (Baba, 2016; Ayman, 2017).

Previously our department has published extensive research on various aspects of prosthetic dentistry ('Evaluation of Corrosive Behavior of Four Nickelchromium Alloys in Artificial Saliva by Cyclic Polarization Test:An in vitro Study', 2017; Ganapathy, Kannan and Venugopalan, 2017; Jain, 2017a, 2017b; Ranganathan, Ganapathy and Jain, 2017; Ariga et al., 2018; Gupta, Ariga and Deogade, 2018; Anbu et al., 2019; Ashok and Ganapathy, 2019; Duraisamy et al., 2019; Varghese, Ramesh and Veeraiyan, 2019), this vast research experience has inspired us to research about Comparison of wear resistance of CAD CAM provisional and heat cure provisional.

\section{MATERIAL AND METHODS}

Sampling: A total of 16 samples, Eight Samples of heat cure provisional restoration (Figure 1) and eight samples of CAD-CAM milled provisional restoration (Figure 2) 
are fabricated. Tooth brushing simulator Toothbrush simulator is a device used to evaluate the wear resistance. In this device, commercially available toothbrushes with medium graded bristles were used. The specimens are fixed in the device and the toothbrush is positioned so that it can simulate brushing. The specimens are fixed in the tooth brush simulator and the brushing simulation is done for the period of 24hrs (Figure 3).

Figure 1: Heat cure provisional sample

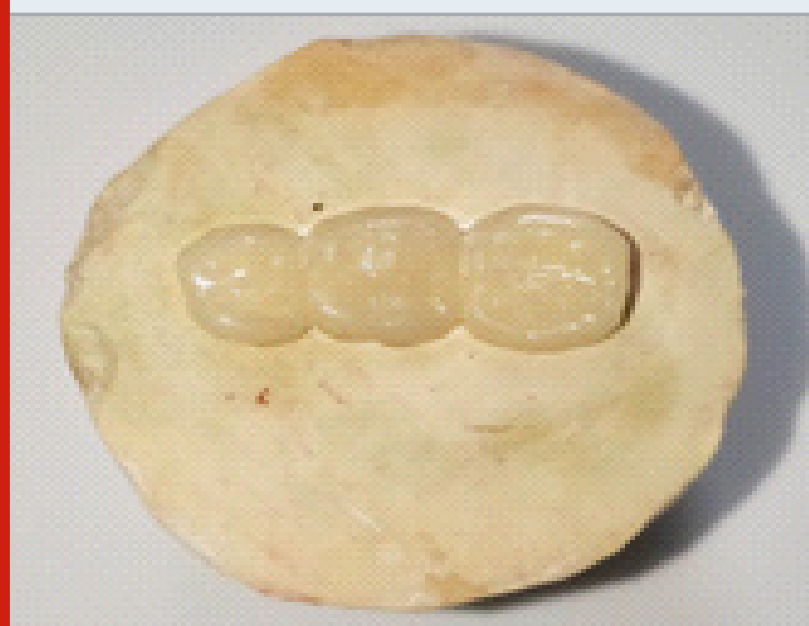

Heat cure FPD

Figure 2: CAD-CAM provisional samples

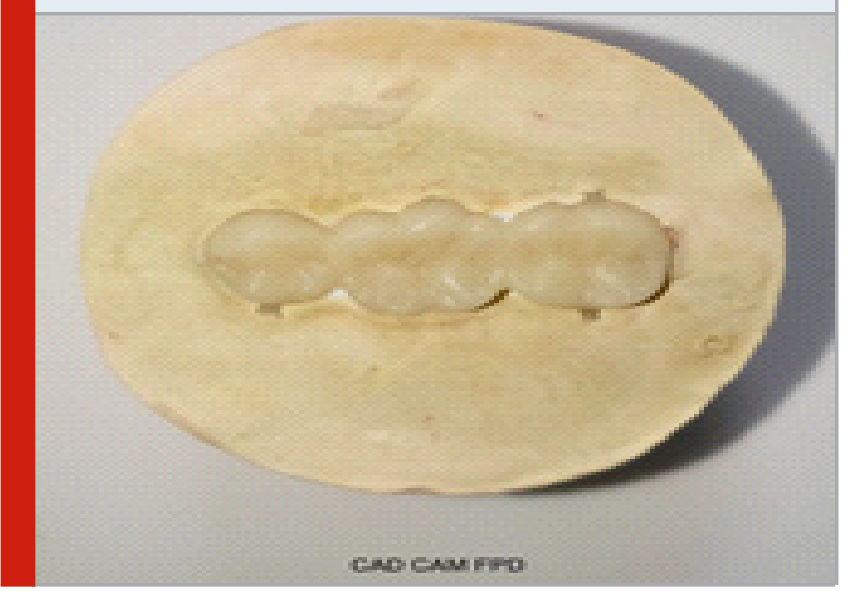

Figure 3: Toothbrush simulator with samples.

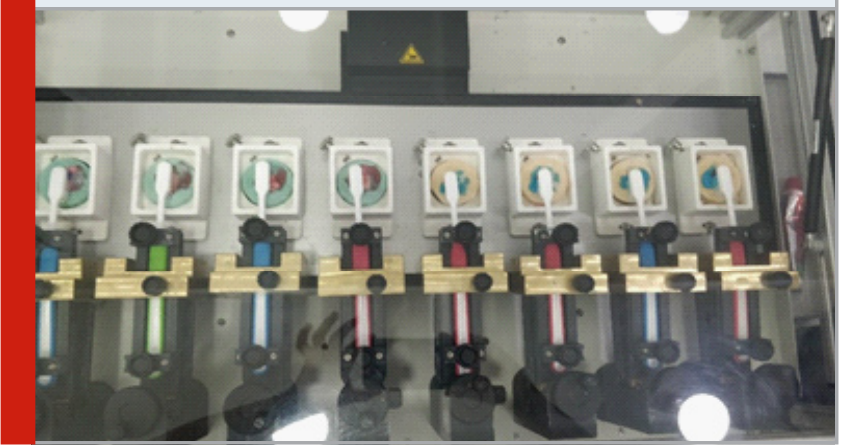

The results are tabulated in excel, Statistical analysis was done using SPSS and one way ANOVA test performed. Statistical analysis done by exporting the data to SPSS for data checking. Data sorted and then represented in frequencies. Descriptive results are presented on tables and graphs.

\section{RESULT AND DISCUSSION}

The wear resistance is less for the Heat cure provisional FPD as compared to the CAD CAM provisional FPD which has more wear resistance. Pre-treatment and post-treatment cuspal thickness shows a vast difference in heat cure provisional as seen in Graph 1. However the pre-treatment and post-treatment cuspal thickness reduction is minimal in cad cam provisional FPD as seen in Graph 2.

Figure 4: Pre treatment and post treatment images of heat cure provisional FPD showing the wear resistance.

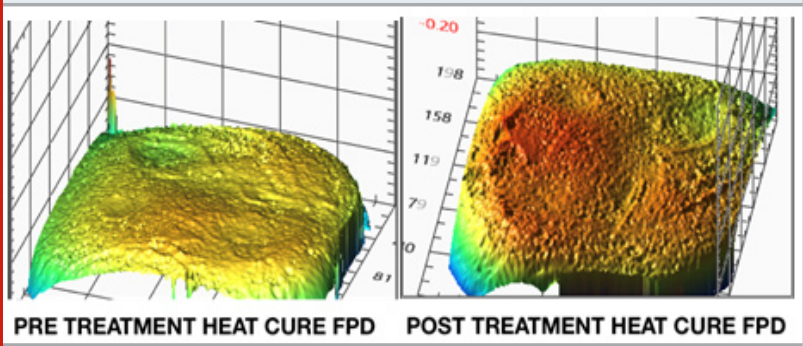

Figure 5: Pre treatment and post treatment images of CADCAM provisional FPD showing the wear resistance.

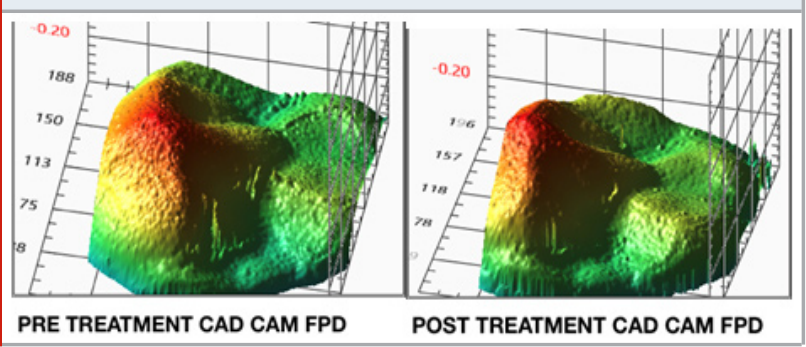

Dental wear is a complex process that is influenced by numerous factors and depends on physiological and pathological mechanisms (Mörmann et al., 2013). Resistance to occlusal wear is an important consideration for the clinical success of oral prosthetic restorations. The wear of the restorative material should match with the wear of natural enamel. Wear of restorative material can cause abnormal loading on the occlusal surface and possibly loss of occlusal vertical dimensions, which can lead to problems, such as temporomandibular joint disorders, masticatory muscle fatigue, changes in mandibular movement path, and esthetic problems (Lambrechts et al., 1989; Mörmann et al., 2013).

To evaluate the wear resistance, several tests have been tried; rotating sliding wear test, two body wear test, three body wear test, and tooth brush simulator. Clinically, although most restorative material or tooth loss may be due to direct contact between teeth, between teeth and 
restorative materials, or between restorative materials, these losses can also be caused by other factors, such as abrasion or erosion (Lim et al., 2002).

Graph 1: This graph shows association between Heat cure provisional restoration and wear resistance where blue color indicates Pretreatment FPD and red color indicates Post treatment FPD. The $\mathrm{X}$ axis represents the Heat cure restoration provisional samples and the $\mathrm{Y}$ axis represents the wear resistance of the samples. The wear resistance is less in heat cure provisional. One way ANOVA test done, $\mathrm{P}<0.001$ for Heat cure provisional restoration indicating statistically significant.

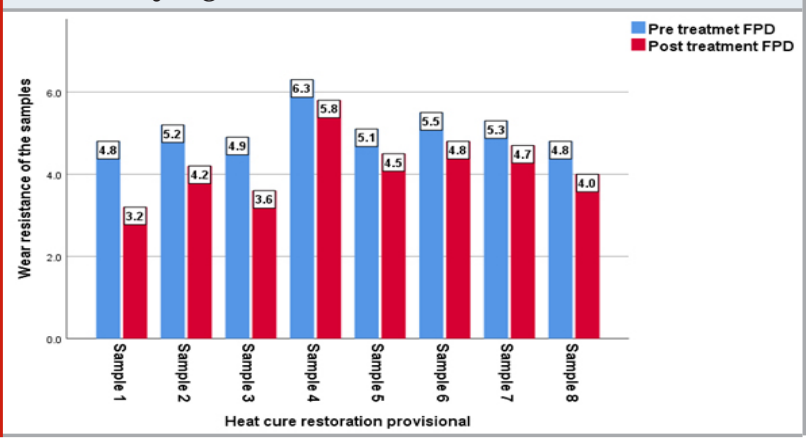

Graph 2: This graph shows association between CAD-CAM restoration provisional and wear resistance where blue color indicates Pretreatment FPD and red color indicates Post treatment FPD. The $\mathrm{X}$ axis represents the CAD-CAM restoration provisional samples and the $\mathrm{Y}$ axis represents the wear resistance of the samples. Wear resistance is more in CAD-CAM provisional restoration.

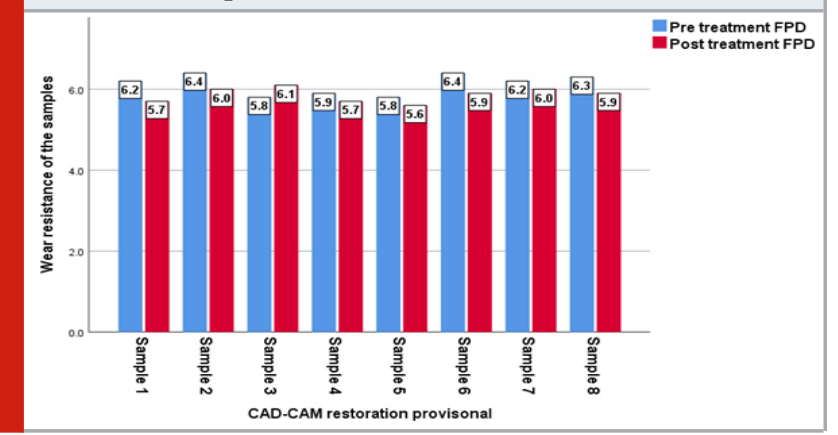

In immediate loading of implant prosthesis and in compromised abutment conditions, the material choice for the fixed restoration is PMMA resin. This PMMA resin should withstand wear for minimum 6 months till permanent restorations are replaced. In this study the wear resistance is tested using the tooth brush simulator for $24 \mathrm{hrs}$. The abrasion wear for a restoration can be 5 minutes per day during brushing. Thus this study shows the abrasion wear value for 9 months. When compared with conventional heat cured acrylic resin, the CDA-CAM resin restoration has better abrasion wear resistance.

\section{CONCLUSION}

CAD-CAM milled PMMA resin has more abrasive wear resistance than the Conventional heat cured acrylic resin. This can due to the less polymerization shrinkage, less residual monomer content, less manual error. The above study concludes that the CAD-CAM milled resin restoration is preferred than conventional heat cured restoration as long term provisional restoration.

\section{ACKNOWLEDGEMENTS}

The study was supported by Saveetha Dental College \& Hospitals, Saveetha Institute of Medical and Technical Sciences, Saveetha University, Chennai.

Conflict of Interest: There are no conflicts of interest as declared by the authors.

\section{REFERENCES}

Ayman, A.-D. (2017) 'The residual monomer content and mechanical properties of CAD $\backslash$ CAM resins used in the fabrication of complete dentures as compared to heat cured resins', Electronic physician, 9(7), pp. 4766-4772.

Baba, N. Z. (2016) 'Materials and Processes for CAD/ CAM Complete Denture Fabrication', Current Oral Health Reports, pp. 203-208. doi: 10.1007/s40496-0160101-3.

Cai, Z.-B. et al. (2011) 'In situ observations of the real-time wear of PMMA flat against steel ball under torsional fretting', Wear, pp. 2242-2251. doi: 10.1016/j. wear.2011.01.069.

Cardoso, M. et al. (2008) 'Influence of application site of provisional cement on the marginal adaptation of provisional crowns', Journal of applied oral science: revista FOB, 16(3), pp. 214-218.

Demir, M. M. et al. (2006) 'PMMA/Zinc Oxide Nanocomposites Prepared by In-Situ Bulk Polymerization', Macromolecular Rapid Communications, pp. 763-770. doi: 10.1002/marc.200500870.

Diaz-Arnold, A. M. et al. (2008) 'Flexural and fatigue strengths of denture base resin', The Journal of Prosthetic Dentistry, pp. 47-51. doi: 10.1016/s00223913(08)60136-5.

$\mathrm{Gu}$, D. et al. (2019) 'Heat treatment to improve the wear resistance of PTFE/PMMA composites', RSC Advances, pp. 22289-22294. doi: 10.1039/c9ra04362d.

Hazelton, L. R. et al. (1995) 'Influence of reinforcement design on the loss of marginal seal of provisional fixed partial dentures', The International journal of prosthodontics, 8(6), pp. 572-579.

John, J. et al. (2015) 'Flexural Properties of Poly(Methyl Methacrylate) Resin Reinforced with Oil Palm Empty Fruit Bunch Fibers: A Preliminary Finding', Journal of Prosthodontics, pp. 233-238. doi: 10.1111/jopr.12191. Keyf, F., Uzun, G. and Mutlu, M. (2003) 'The effects of HEMA-monomer and air atmosphere treatment of glass fibre on the transverse strength of a provisional fixed partial denture resin', Journal of Oral Rehabilitation, pp. 1142-1148. doi: 10.1046/j.1365-2842.2003.01059.x. 
Kim, S.-H. and Watts, D. C. (2004) 'Effect of glass-fiber reinforcement and water storage on fracture toughness (KIC) of polymer-based provisional crown and FPD materials', The International journal of prosthodontics, 17(3), pp. 318-322.

Lambrechts, P. et al. (1989) 'Quantitative in vivo wear of human enamel', Journal of dental research, 68(12), pp. 1752-1754.

Lewinstein, I. et al. (2003) 'Retention, marginal leakage, and cement solubility of provisional crowns cemented with temporary cement containing stannous fluoride', The International journal of prosthodontics, 16(2), pp. 189-193.

Lim, B. S. et al. (2002) 'Effect of filler fraction and filler surface treatment on wear of microfilled composites', Dental materials: official publication of the Academy of Dental Materials, 18(1), pp. 1-11.

Mörmann, W. H. et al. (2013) 'Wear characteristics of current aesthetic dental restorative CAD/CAM materials: two-body wear, gloss retention, roughness and Martens hardness', Journal of the mechanical behavior of biomedical materials, 20, pp. 113-125.

Ovsianikov, A. et al. (2011) 'Laser Fabrication of Three-Dimensional CAD Scaffolds from Photosensitive Gelatin for Applications in Tissue Engineering', Biomacromolecules, pp. 851-858. doi: 10.1021/ bm 1015305.

Ruyter, I. E., Nilner, K. and Moller, B. (1987) 'Color stability of dental composite resin materials for crown and bridge veneers', Dental materials: official publication of the Academy of Dental Materials, 3(5), pp. 246-251.

Scotti, R., Mascellani, S. C. and Forniti, F. (1997) 'The in vitro color stability of acrylic resins for provisional restorations', The International journal of prosthodontics, 10(2), pp. 164-168.

Vallittu, P. K. (1993) 'Comparison of two different silane compounds used for improving adhesion between fibres and acrylic denture base material', Journal of oral rehabilitation, 20(5), pp. 533-539.

Vallittu, P. K. (1999) 'Flexural properties of acrylic resin polymers reinforced with unidirectional and woven glass fibers', The Journal of prosthetic dentistry, 81(3), pp. 318-326.

Vallittu, P. K., Ruyter, I. E. and Ekstrand, K. (1998) 'Effect of water storage on the flexural properties of E-glass and silica fiber acrylic resin composite', The International journal of prosthodontics, 11(4), pp. 340-350.

Wassell, R. W. et al. (2002) 'Crowns and other extra-coronal restorations: Provisional restorations', British Dental Journal, pp. 619-630. doi: 10.1038/ sj.bdj.4801443. 